\title{
Genomic characterization of fecal microbial communities in severely sick humans and broiler chickens using next generation sequencing
}

\section{Ome Kalsoom Afridi}

Kyungpook National University

Johar Ali ( $\sim$ Johar.ali1@rmi.edu.pk)

https://orcid.org/0000-0003-1062-3204

Jeong Ho Chang

Kyungpook National University

\section{Research article}

Keywords: comparative microbiome profiling, next-generation sequencing, microbial diversity, microbial association, dysbiosis, shotgun metagenomics, fecal microbiota, commercial broilers, microbial communities

Posted Date: February 5th, 2020

DOI: https://doi.org/10.21203/rs.2.22699/v1

License: (c) (i) This work is licensed under a Creative Commons Attribution 4.0 International License. Read Full License 


\section{Abstract}

Background: Microbiota plays an important role in food safety and its alteration poses a serious threat to humans. Comparative microbiome profiling using next-generation sequencing (NGS) enabled the understanding of microbial diversity and similarity between different species. In this study, we used NGS to profile the fecal microbiota of sick human and broiler chickens. A total of 26 fecal samples were collected from severely sick human subjects $(n=13)$ and broiler chickens $(n=13)$ with similar symptoms.

Results: The total number of microbial species detected in broiler chickens fecal microbiota was higher than that of humans. Phylum Proteobacteria was the most abundant in both human and broiler chickens fecal microbiota while Tenericutes was found to be least abundant in both species. Phylum Actinobacteria was found only in the human fecal microbiota. In both humans and broiler chickens, E.coli was found to be phylogenetically related suggesting a microbial association between both species.

Conclusion: NGS based taxonomic profiling revealed the association of microbial dysbiosis with extreme sickness in both humans and broiler chickens. The dominance of phylum Proteobacteria in both the species ascertains their altered gut microbiota. Both human and broiler chickens microbial communities were found to be genetically related indicating horizontal transfer of microbes between the two species.

\section{Background}

With the advent of next-generation sequencing technology (NGS), microbial communities within the gut microbiome of human and farm animals have been studied extensively [1]. Until now, microbial communities within the gut of human and various animals were studied using culture-dependent methodologies which hampered the growth of anaerobic bacteria [2-3]. Previously, the majority of intestinal bacteria were considered 'unculturable' using standard laboratory culture conditions [4]. The normal human gut microbial communities are dominated by three anaerobic bacteria phyla including Bacteriodes, Firmicutes, and Actinobacteria [5]. Similarly, Firmicutes, Proteobacteria, and Bacteroidetes have been identified as the three dominated bacterial phyla within the poultry gut microbial communities [6]. The other important taxonomic groups present within the gut-microbiome of human are Proteobacteria, Actinobacteria, Fusobacteria, Verrucomicrobia, methanogenic archaea, and various other diverse eukaryotic communities such as protists and fungi [7]. A number of microbes transiently colonize gut conditions. Until now, the important roles of the human microbiome in health and diseases were not clear due to the limitations of standard culture-based techniques [8]. However, high throughput sequencing fast-tracked gut microbiome research [9]. Currently, 16S rDNA amplicon and shotgun metagenomic sequencing are the two common approaches used to address different aspects of gut microbiota in various pathological conditions including inflammatory bowel disease, diabetes, and obesity [10]. In comparison with 16S rDNA amplicon sequencing, shotgun metagenomic offers greater sensitivity, specificity, microbial abundance estimation, and greater resolution of microbes up to the species level [11]. Evidence support the fact that various pathogenic microbes can be transmitted from animals to humans or from humans to animals either through direct contact or while living with livestock 
in close contact and it has been exemplified by a recent report indicating the transmission of antibioticresistant bacteria including methicillin-resistant Staphylococcus aureus (MRSA), multidrugresistant Staphylococcus aureus, and various members of extended-spectrum beta-lactamase (ESBL) producing Enterobacteriaceae [12-14]. Currently, foodborne infections pose a serious health threat to human life and such infections are transmitting from food-producing animals to humans via the food chain [15]. Among the various foodborne pathogens, Salmonella, Campylobacter, Listeria, Escherichia, Shigella, Yersinia, and Vibrio are the major microbial genera causing significant morbidity and mortality across the globe $[16,17]$. The human gut is the reservoir of trillions of microbes comprising of not only normal flora but a variety of pathogenic bacteria and the great majority of these pathogens are zoonotic [18]. Broiler chickens are considered as an economic alternative of red-meat in Pakistan and are consumed heavily across the country [19]. Antibiotics as growth promoters are banned in various developed countries [20] however, in Pakistan, various antibiotics are still frequently used as feed additives [21]. Recent chicken microbiome studies documented that multiple factors including feed alterations, the composition of litter, antibiotics, the addition of probiotics to the feed, various diseases and stress are linked with alteration of their gut-microbiome [22, 23]. According to the One Health approach, the transmission of various pathogenic microbes among different hosts is due to the existence of an ecological relationship between humans, animals, and environmental health [24]. Investigation of microbial transfer between humans, various animals, and the environment is needed while applying One Health concept to the microbiome-based studies [25, 26]. Understanding the association of various microbiomes between different species and their environment can reveal novel approaches related to diagnosis, treatment, and intervention of several deadly diseases [27]. Keeping in view the One Health approach, we attempted to profile the fecal microbiota of human (HS) and broiler chickens (BC) using NGS shotgun metagenomic sequencing. Besides comparative microbial profiling, one of our objectives was to ascertain the phylogenetic relatedness of bacterial species between humans and poultry.

\section{Results}

\section{Selection of samples with similar symptoms for sequencing}

On the basis of similar symptoms, we selected 26 samples (13 each) out of 1000 screened samples for shotgun metagenomic sequencing of $\mathrm{HC}$ and $\mathrm{BC}$ fecal microbiota.

\section{Microbial taxonomy}

Microbial profiling of HS and BC fecal samples was carried out using MEGAN6 software [67]. The microbial profiles of dominated phyla, families, and genera of HS and BC fecal samples are shown in Figures 3 and 4, respectively.

\section{Microbial profiling at the phylum level}

In total, 5 phyla were identified in HS fecal samples namely Proteobacteria, Bacteroidetes, Firmicutes, Actinobacteria, and Tenericutes. Conversely, 4 phyla were identified in BC fecal samples namely 
Proteobacteria, Firmicutes, Bacteroidetes, and Tenericutes. The fecal microbial profile of HS samples showed the presence of Actinobacteria whereas this specific phylum was not found in BC fecal samples. Among all these phyla, Proteobacteria was the most dominated phylum in both HS and BC fecal samples accounted for $45.6 \%$ and $38.9 \%$, respectively. In HS samples, the second most abundant phylum was Bacteroidetes (36.6\%), followed by Firmicutes (12.5\%), Actinobacteria (5\%), and Tenericutes (0.3\%). In BC fecal samples, the second most abundant phylum was Firmicutes (36.4\%), followed by Bacteroidetes (15.8\%) and Tenericutes (8.9\%).

\section{Microbial profiling at the family level}

In both $\mathrm{HS}$ and $\mathrm{BC}$ fecal samples, 10 microbial families were found to be the most abundant. Families dominated in HS fecal microbiota were Enterobacteriaceae, Bacteroidaceae, Ruminococcaceae, Clostridiaceae, Bifidobacteriaceae, Enterococcaceae, Selenomonadaceae, Sphingobacteriaceae, Streptococcaceae and Aeromonadaceae. In contrast to the HS fecal microbiota, families dominated in BC fecal samples were Enterobacteriaceae, Bacteroidaceae, Clostridiaceae, Lactobacillaceae, Pseudomonadaceae, Acholeplasmataceae, Staphylococcaceae, Lachnospiraceae, Ruminococcaceae, and Caulobacteraceae. Families specifically found only in HS fecal microbiota were Bifidobacteriaceae, Enterococcaceae, Selenomonadaceae, Sphingobacteriaceae, Streptococcaceae, and Aeromonadaceae whereas Lactobacillaceae, Pseudomonadaceae, Acholeplasmataceae, Staphylococcaceae, Lachnospiraceae, and Caulobacteraceae dominated only in BC fecal microbiota. Among all these families, Enterobacteriaceae was the most abundant in both HS and BC fecal samples, which accounted for $40 \%$ and $24.1 \%$ respectively. Similarly, family Bacteroidaceae was the second most abundant family in both HS and BC fecal samples, which accounted for $32 \%$ and $16.4 \%$ respectively. The third most abundant family in HS fecal microbiota was Ruminococcaceae (8.8\%) followed by Clostridiaceae (5.1\%), Bifidobacteriaceae (4.4\%), Enterococcaceae (3.3\%), Selenomonadaceae (1.8\%), Sphingobacteriaceae $(1.3 \%)$, Streptococcaceae $(0.9 \%)$ and Aeromonadaceae $(0.7 \%)$. Conversely, the third most abundant family in BC fecal samples was Clostridiaceae (11.7\%) followed by Lactobacillaceae (11.4\%), Pseudomonadaceae (10.9\%), Acholeplasmataceae (8\%), Staphylococcaceae(7.3\%), Lachnospiraceae (5.2\%), Ruminococcaceae (4.7\%) and Caulobacteraceae (0.3\%). Overall, 6 families were found unique in both HS and BC fecal samples.

\section{Microbial profiling at the genus level}

Microbial profiling revealed 10 abundant genera in both HS and BC fecal samples. Those included Bacteroides, Escherichia, Enterobacter, Salmonella, Faecalibacterium, Ruminococcus, Alistipes, Bifidobacterium, Enterococcus, Coprococcus, Pseudomonas, Lactobacillus, Faecalibacterium, Phytoplasma and Staphylococcus. Among these, genus Bifidobacterium, Enterococcus and Coprococcus dominated specifically only in HS samples while Pseudomonas, Lactobacillus, Faecalibacterium, Phytoplasma, and Staphylococcus dominated only in BC fecal samples. The most abundant genera in HS samples were Bacteroides (22.9\%) followed by Escherichia (20.1\%), Enterobacter (9.7\%), Salmonella (9.1\%), Faecalibacterium (7.1\%), Ruminococcus (6.1\%), Alistipes (4.8\%), Bifidobacterium (3.1\%), 
Enterococcus (2.9\%) and Coprococcus (2.7\%). In contrast, the most abundant genera found in BC fecal microbiota were Escherichia (15.9\%) followed by Alistipes (13.5\%), Bacteroides (10.9\%), Pseudomonas (10.9\%), Lactobacillus (10\%), Faecalibacterium (10.3\%), Phytoplasma (8\%), Ruminococcus (7.4\%), Staphylococcus (6.9\%) and Salmonella (6.4\%).

\section{Microbial profiling at the species level}

The various abundant and common species found in HS and BC fecal samples are shown in Figures 5, 6 and 7 respectively. In total, 37 species were found in HS fecal microbiota while 58 species were found in BC fecal samples. Among the various species, E.coli, Salmonella enterica, Klebsiella oxytoca, Klebsiella aerogenes, Klebsiella pneumoniae, Raoultella ornithinolytica, Bacteroides fragilis, Bacteroides thetaiotaomicron, Butyrate producing bacterium SS3/4, Ruminococcus bromii, Ruminococcus champanellensis, Ruminococcus torques, Faecalibacterium prausnitzii, Alistipes shahii, Arsenophonus nasoniae, Shigella flexneri, and Enterobacter cloacae were the unique species found HS fecal samples. In contrast, species found only BC fecal samples were Shigella dysentriae, Shigella sp.SF-2015, Yersinia enterocolitica, Escherichia fergusonii, Blautia obeum A2-162, Butyrivibrio fibrisolvens, Ralstonia solanacearum, Marinobacter adhaerens, Achromobacter xylosoxidans, Pseudomonas fluorescens, Pseudomonas stutzeri, Pseudomonas putida, Pseudomonas aeruginosa, Staphylococcus xylosus, Staphylococcus aureus, Staphylococcus saprophyticus, Staphylococcus epidermidis, Staphylococcus warneri, Staphylococcus pettenkoferi, Solibacillus silvestris, Halobacillu shalophilus, Caulobacter vibrioides, Bacillus subtilis, Lactobacillus salivarius, Lactobacillus johnsonii, Lactobacillus helveticus.

\section{Phylogenetic analysis}

Genetic analysis of E.coli found in both HS and BC fecal samples was carried out against the reference strain E.coli K-12 substr. MG1655 (NC 000913.3) on the basis of sequence similarity. E.coli dominated in BC fecal microbial profile was found to be $99.48 \%$ similar to the reference E.coli strain. Conversely, E.coli dominated in $\mathrm{HS}$ fecal samples was found to be $98.2 \%$ similar to the reference strain. Upon comparing the sequence similarity of E.coli dominated in the $\mathrm{HS}$ and $\mathrm{BC}$ fecal samples with respect to each other, the similarity was found to be $98 \%$ (Figure 8 ).

\section{Discussion}

In this study, we used NGS shotgun metagenomics to profile HS and BC fecal microbiota of severely sick $\mathrm{HS}$ and $\mathrm{BC}$ with similar symptoms. Phylogenetic analysis was performed to investigate the bacterial association between humans and poultry. Metagenomic profiling of HS fecal microbiota revealed a predominance of phylum Proteobacteria, Bacteroidetes, Firmicutes, Actinobacteria while Tenericutes was the least dominant group. Similarly, BC fecal microbiota was dominated by Proteobacteria, Firmicutes, Bacteroidetes while Tenericutes constitute the least abundant group. We observed that Proteobacteriawas dominantly present in both HS and BC fecal microbiota. The higher abundance of Proteobacteria in this study reflects the state of the altered gut microbiome of both humans and poultry $[28,29]$. Our findings are in line with a previous study that reported a significantly higher abundance of 
phylum Proteobacteria in the hospitalized Israeli population [30]. Similarly, another study found that the distribution of various bacterial phyla in pigs was altered after 14 days of antibiotics intake with a considerable increase in Proteobacteria in comparison with the group raised without antibiotics using the approach of 16S rDNA amplicon sequencing [31]. Furthermore, a recent study found a significantly higher percentage of Proteobacteria in fecal samples of both layers and BC speculating that antibiotic intake might be the contributing factor for the alteration of gut microbiota [32]. The predominance of Proteobacteria has been documented as a diagnostic marker of various diseases and dysbiosis [33]. An altered gut microbiome is termed as dysbiosis which may lead to various pathological conditions such as reducing the barrier function of the intestine, reduced nutrient digestive potential, increased risk of bacterial translocation and various inflammatory conditions [34]. In addition, the predominance of Proteobacteria has been reported to be associated with the emergence of opportunistic pathogens [35, 36].

During the taxonomic profiling of the samples, we observed that phylum Actinobacteria was present only in HS microbiota. The complete loss of Actinobacteria in BC fecal microbiota can be attributed to the high usage of antibiotics as growth promoters in BC feed. Our suspicion is supported by a recent report indicating a decrease in Actinobacteria in chicken fed with antibiotics as growth promoters [37, 38]. In our study, we observed that phylum Tenericutes was the least abundant in both HS and BC fecal microbiota. This could be explained by a recent report indicating that antibiotic treatment drastically affected the abundance of Tenericutes [39].

Looking at bacterial class level diversity, we found 10 different classes in HS fecal whereas only 6 classes were detected in BC fecal samples (Fig. 3 and 4). Various factors are reported to affect the diversity of gut microbiota including age, intestinal transit time, diet and use of antibiotics as growth promoters in chicken feed [40-42]. Mortality at an early age can possibly be a reason for lower microbial diversity in $\mathrm{BC}$ fecal microbiota. A recent report demonstrated the enrichment of intestinal microbial diversity with increasing age in chickens [6].

We found common useful and pathogenic genera between HS and BC fecal samples. Useful common genera in HS and BC fecal samples were Bacteroides, Faecalibacterium, Alistipes, and Ruminococcus while Escherichia and Salmonella were the common pathogenic genera. Our result of a greater abundance of Bacteroides and Escherichia in HS microbiota is in agreement with a previous report indicating a higher abundance of Escherichia and Bacteroidetes in elderly subjects indicating gut dysbiosis [43]. The results of this study based on the genera distribution of BC fecal microbiota are not in agreement with the previous studies [44-47]. The variation of our results from the previous studies could possibly be explained by the fact that their studies were based on $16 \mathrm{~S}$ rDNA amplicon sequencing using healthy chickens raised under various conditions. In our study, the presence of a higher abundance of pathogenic genera in both HS and BC samples is in line with a recent report indicating that a higher abundance of the particular pathogen within the intestinal communities causes greater liability to be affected by those particular pathogens. Furthermore, a higher abundance of commensal E. coli was attributed to an increased level of Salmonella enterica infections [48]. 
A number of common useful and pathogenic bacterial species were identified at the species level in HS and BC fecal microbiota. Useful common species in HS and BC were Bacteroides fragilis, Bacteroides thetaiotaomicron, Butyrate producing bacterium SS3/4, Ruminococcusbromii, Ruminococcus champanellensis, Ruminococcus torques, Faecalibacterium prausnitzii, and Alistipes shahii while common pathogenic bacteria were E.coli, Salmonella enterica, Klebsiella oxytoca, Klebsiella aerogenes, Klebsiella pneumoniae, Raoultella ornithinolytica, Arsenophonus nasoniae, Shigella flexneri, and Enterobacter cloacae.

The various pathogenic bacterial species identified in $\mathrm{BC}$ fecal microbiota have been reported to be zoonotic pathogens transmitted to humans through contaminated food [49-51]. Humans acquire the majority of foodborne infections upon direct or indirect exposure to contaminated food from a variety of host species including poultry, dogs, cats and livestock [52]. The co-dominance of various foodborne pathogens such as E.coli and Salmonella enteric in both HS and BC fecal microbiota indicates a possible route through which these pathogenic bacteria are transferred between the two species. Among the different pathogenic bacteria, E.coli has been considered as an important source of serious infections in both humans and poultry [53]. E.coli is the causative agent of infections designated as extraintestinal pathogenic E. coli (ExPEC) [54]. Among the various forms of ExPEC, avian-pathogenic E.coli (APEC) causes severe poultry infections collectively called as avian Colibacillosis [55]. Similarly, the intestinal tract of humans is considered as the main reservoir of ExPEC, however, multiple recent reports have indicated that BC can act as an external source of ExPEC transmitted to humans through the food chain [56]. Our results based on phylogenetic analysis revealed that E.coli from BC and HS fecal samples was $99.48 \%$ and $98.2 \%$ similar to the reference strain, respectively. Furthermore, we found that E.coli from HS and BC fecal microbiota had $98 \%$ identity to each other. In the present study, similarity found through phylogenetic analysis between E.coli from HS and BC fecal microbiota might reflect the transmission of pathogenic E.coli from poultry to humans through the food chain. Our suspicion of E.coli transmission from poultry to humans is supported by few studies indicating that avian pathogenic APEC and human ExPEC strains are phylogenetically related [57-58].

\section{Conclusion}

The alteration of gut microbiota with various factors is now a well-established fact. In this study, we compared the gut microbiome of severely sick humans and poultry using NGS. Our findings demonstrate the identification of Proteobacteria to be the most abundant phylum in both HS and BC fecal samples indicating an altered gut microbiome. Phylum Actinobacteria was found only in HS fecal samples providing a point of difference between the two species. Moreover, Bacteroides and Escherichia were the most abundant genera in HS and BC fecal samples respectively. Our findings indicate a higher number of bacterial species in $\mathrm{BC}$ than that of $\mathrm{HS}$ fecal samples. The presence of various pathogenic bacterial species in HS and BC fecal samples reflects their altered gut microbial communities. Furthermore, this study found that human and poultry E.coli are phylogenetically related indicating horizontal transfer of microbes between the two species. 


\section{Methods}

Fecal samples were used for gut microbiome profiling of human and broiler chickens.

\section{Collection of fecal samples}

Initially, we screened 1000 samples for both human and poultry using the records of Microbiology Laboratory of Hayatabad Medical complex, and Veterinary Research Institute, Peshawar Pakistan. Fecal samples $(n=26)$ were collected from the severely sick human subjects $(n=13$, aged 45 years $)$ and broiler chickens ( $n=13$, aged $4-5$ weeks) with similar symptoms under aseptic measures. Fecal samples were transported to the laboratory on ice and were mixed thoroughly using a spatula. Aliquots of $0.2 \mathrm{~g}$ were made and stored at $-80^{\circ} \mathrm{C}$.

\section{DNA extraction}

DNA from HS and BC fecal samples were extracted using PureLink ${ }^{T M}$ Microbiome DNA Purification Kit (Invitrogen, ThermoFisher Scientific; Cat. no. A29790) following manufacturer's instruction with modifications made to optimize the protocol according to the simple laboratory benchtop instruments (unpublished data). Genomic DNA concentration was determined using Qubit fluorometer following manufacturer's instructions (Qubit ${ }^{\mathrm{TM}}$ fluorometer, Invitrogen, CA 92008, USA) [59].

\section{NGS library preparation}

DNA was used to prepare NGS library using Illumina ${ }^{\circledR}$ Nextera XT DNA Library Preparation Kit (FC-1311096, Illumina Inc., CA, USA) and Nextera XT Index Kit v2 Set A (FC-131-2001, Illumina Inc., CA, USA) by following manufacturer's instructions [60]. The generated DNA libraries were pooled and loaded onto the flow cell. Paired-end sequencing (2x150 bp) was performed using Illumina MiSeq (Illumina Inc., CA, USA) [61]. The reagent cartridge used for sequencing was 300 cycle V2 MiSeq reagent kit(MS-103-1002, Illumina Inc., CA, USA).

\section{Sequencing data analysis}

\section{Paired-end sequencing generated FASTQ files that were analyzed using various publicly available bioinformatics softwares. NGS sequenced data were analyzed through multiple steps as follows:}

1. All FASTQ data were de-multiplexed using the CASAVA 8.2 package [62].

2. By using our custom data analysis pipeline, all the samples were analyzed for microbial characterization (Fig. 1). 
3. Raw data comprising of low quality reads, adapters and technical biases were removed using Trimmomatic 0.36 [63].

4. All the microbial reads were decontaminated from human DNA using a computational tool Knead Data 0.6.1 [64].

5. De-novo assembly of microbes was carried out by using SPAdes assembler v.5.0 [65].

6. The microbial profiles were further aligned against NCBI's non-redundant protein reference database (NCBI-NR) using DIAMOND aligner v.0.9.25 [66].

7. DIAMOND blast results were interpreted using MEtaGenome ANalyzer(MEGAN6) software for the taxonomic classification of microbes [67].

\section{Genetic analysis}

For genetic analysis, FASTQ files of various bacterial strains detected within the fecal samples of HS and BC were aligned against the reference genome. Samtools mpileup v.1.9 was used to generate FASTA files [68]. Using ClustalW, all the FASTA files were aligned against the reference genome and a phylogenic tree was constructed using the BioNJ distance algorithm $[69,70]$. The complete bioinformatics workflow of genetic analysis is shown in Fig. 2.

\section{Visualization of microbial profiles}

Microbial community abundance was visualized using Pavain to generate cladograms for various taxonomic groups while hclust2 was used to generate heatmaps based on Bray-Curtis dissimilarity for sample clustering [71-72].

\section{Declarations}

\section{Ethics approval and consent to participate}

Ethical approval was obtained from the Rehman Medical Institute-Research Ethics Committee (Ref: RMI/RMI-REC/Approval/33) Peshawar, Pakistan. Informed consent was obtained from the all patients who participated in this study.

\section{Consent for publication}

Not applicable

\section{Availability of data and materials}

The datasets analyzed during the current study are available from the corresponding author on reasonable request.

\section{Competing interests}


The authors declare that they have no competing interests.

\section{Funding}

This research was supported by funds from the Basic Science Research Program of the National Research Foundation of Korea, funded by the Ministry of Science and ICT (grand No. NRF-2019 R1A2C4069796 to JHC).

\section{Authors' contributions}

OKA performed experiments, analyzed data, wrote the paper. JA co-supervised and designed the study, provided advice in data analysis, critically discussed results and edited paper. JHC supervised the study, provided advice in study design and co-edited the paper.

\section{Acknowledgments}

All the authors acknowledge Syed Adnan Haider, Bibi Sabiha and Hanif Ullah Jan for their outstanding technical support.

\section{References}

1. Ji B, Nielsen J. From next-generation sequencing to systematic modeling of the gut microbiome. Front Genet. 2015; 6:219.

2. Shang Y, Kumar S, Oakley B, Kim WK. Chicken gut microbiota: importance and detection technology. Front. Vet. Sci. 2018; 5:254.

3. Lagier JC, Million M, Hugon P, Armougom F, Raoult D. Human gut microbiota: repertoire and variations. Front. Cell. Infect. Microbiol. 2012; 2:136.

4. Browne HP, Forster SC, Anonye BO, Kumar N, Neville BA, Stares MD, et al. Culturing of 'unculturable' human microbiota reveals novel taxa and extensive sporulation. Nature. 2016; 533(7604):543.

5. Lozupone CA, Stombaugh JI, Gordon JI, Jansson JK, Knight R. Diversity, stability and resilience of the human gut microbiota. Nature. 2012; 489(7415):220.

6. Shaufi MA, Sieo CC, Chong CW, Gan HM, Ho YW. Deciphering chicken gut microbial dynamics based on high-throughput 16S rRNA metagenomics analyses. Gut Pathog. 2015; 7(1):4.

7. Tap J, Mondot S, Levenez F, Pelletier E, Caron C, Furet JP, et al. Towards the human intestinal microbiota phylogenetic core. Environ Microbiol. 2009; 11(10):2574-84.

8. Gu W, Miller S, Chiu CY. Clinical metagenomic next-generation sequencing for pathogen detection. Annu Rev Pathol. 2019; 14:319-38.

9. Methe BA, Nelson KE, Pop M, Creasy HH, Giglio MG, Huttenhower C, et al. A framework for human microbiome research. Nature. 2012; 486(7402):215. 
10. Malla MA, Dubey A, Kumar A, Yadav S, Hashem A, Abd_Allah EF. Exploring the human microbiome: The potential future role of next-generation sequencing in disease diagnosis and treatment. Front. Immunol. 2018; 9.

11. Grutzke J, Malorny B, Hammerl JA, Busch A, Tausch SH, Tomaso H, et al. Fishing in the soupPathogen detection in food safety using Metabarcoding and Metagenomic sequencing. Front. Microbiol. 2019; 10:1805.

12. Liu W, Liu Z, Yao Z, Fan Y, Ye X, Chen S. The prevalence and influencing factors of methicillinresistant Staphylococcus aureus carriage in people in contact with livestock: A systematic review. Am J Infect Control. 2015; 43(5):469-75.

13. Frana TS, Beahm AR, Hanson BM, Kinyon JM, Layman LL, Karriker LA, et al. Isolation and characterization of methicillin-resistant Staphylococcus aureus from pork farms and visiting veterinary students. PLoS One. 2013; 8(1):e53738.

14. von Salviati C, Laube H, Guerra B, Roesler U, Friese A. Emission of ESBL/AmpC-producing Escherichia coli from pig fattening farms to surrounding areas. Vet Microbiol. 2015; 175(1):77-84.

15. Lee JH. Methicillin (oxacillin)-resistant Staphylococcus aureus strains isolated from major food animals and their potential transmission to humans. Appl. Environ. Microbiol. 2003; 69(11):6489-94.

16. Heredia N, Garcia S. Animals as sources of food-borne pathogens: A review. Animal nutrition. 2018; 4(3):250-5.

17. Kirk MD, Pires SM, Black RE, Caipo M, Crump JA, Devleesschauwer B, et al. World Health Organization estimates of the global and regional disease burden of 22 foodborne bacterial, protozoal, and viral diseases, 2010: a data synthesis. PLoS Med. 2015; 12(12):e1001921.

18. Guenther S, Wuttke J, Bethe A, Vojtech J, Schaufler K, Semmler T, et al. Is fecal carriage of extendedspectrum- $\beta$-lactamase-producing Escherichia coli in urban rats a risk for public health?. Antimicrob. Agents Chemother. 2013; 57(5):2424-5.

19. Ahmad F, Haq AU, Ashraf M, Abbas G, Siddiqui MZ. Effect of different light intensities on the production performance of broiler chickens. Pak. Vet. J. 2011;31(3):203-6.

20. Roth N, Kasbohrer A, Mayrhofer S, Zitz U, Hofacre C, Domig KJ. The application of antibiotics in broiler production and the resulting antibiotic resistance in Escherichia coli: A global overview. Poult Sci. 2018; 98(4):1791-804.

21. Ahmad S, Rehman R, Haider S, Batool Z, Ahmed F, Ahmed SB, et al. Quantitative and qualitative assessment of additives present in broiler chicken feed and meat and their implications for human health. JPMA 2018; 68(6):876-81.

22. Clavijo V, Florez MJ. The gastrointestinal microbiome and its association with the control of pathogens in broiler chicken production: a review. Poultry Sci. 2017; 97(3):1006-21.

23. Cressman MD, Yu Z, Nelson MC, Moeller SJ, Lilburn MS, Zerby HN. Interrelations between the microbiotas in the litter and in the intestines of commercial broiler chickens. Appl. Environ. Microbiol. 2010; 76(19):6572-82. 
24. Davis MF, Rankin SC, Schurer JM, Cole S, Conti L, Rabinowitz P, et al. Checklist for One Health epidemiological reporting of evidence (COHERE). One Health. 2017; 4:14-21.

25. Flandroy L, Poutahidis T, Berg G, Clarke G, Dao MC, Decaestecker E, et al. The impact of human activities and lifestyles on the interlinked microbiota and health of humans and of ecosystems. Sci Total Environ. 2018; 627:1018-38.

26. Song SJ, Lauber C, Costello EK, Lozupone CA, Humphrey G, Berg-Lyons D, et al. Cohabiting family members share microbiota with one another and with their dogs. 2013; 2:e00458.

27. Adams RI, Bateman AC, Bik HM, Meadow JF. Microbiota of the indoor environment: a meta-analysis. Microbiome. 2015; 3(1):49.

28. Hughes ER, Winter MG, Duerkop BA, Spiga L, de Carvalho TF, Zhu W, et al. Microbial respiration and formate oxidation as metabolic signatures of inflammation-associated dysbiosis. Cell Host Microbe. 2017; 21(2):208-19.

29. Litvak Y, Byndloss MX, Tsolis RM, Baumler AJ. Dysbiotic Proteobacteria expansion: a microbial signature of epithelial dysfunction. Curr Opin Microbiol. 2017; 39:1-6.

30. Braun T, Di Segni A, BenShoshan M, Asaf R, Squires JE, Barhom SF, et al. Fecal microbial characterization of hospitalized patients with suspected infectious diarrhea shows significant dysbiosis. Sci Rep. 2017; 7(1):1088.

31. Looft T, Johnson TA, Allen HK, Bayles DO, Alt DP, Stedtfeld RD, et al. In-feed antibiotic effects on the swine intestinal microbiome. Proc Natl Acad Sci U S A. 2012; 109(5):1691-6.

32. Tong P, Ji X, Chen L, Liu J, Xu L, Zhu L, et al. Metagenome analysis of antibiotic resistance genes in fecal microbiota of chickens. Agri Gene. 2017; 5:1-6.

33. Shin NR, Whon TW, Bae JW. Proteobacteria: microbial signature of dysbiosis in gut microbiota. Trends Biotechnol. 2015; 33(9):496-503.

34. Teirlynck E, Gussem MD, Dewulf J, Haesebrouck F, Ducatelle R, Van Immerseel F. Morphometric evaluation of "dysbacteriosis" in broilers. Avian Pathol. 2011; 40(2):139-44.

35. Le Roy Cl, Woodward MJ, Ellis RJ, La Ragione RM, Claus SP. Antibiotic treatment triggers gut dysbiosis and modulates metabolism in a chicken model of gastro-intestinal infection. BMC Vet Res. $2019 ; 15(1): 37$.

36. Cox LM, Yamanishi S, Sohn J, Alekseyenko AV, Leung JM, Cho I, et al. Altering the intestinal microbiota during a critical developmental window has lasting metabolic consequences. Cell. 2014; 158(4):705-21.

37. Wise MG, Siragusa GR. Quantitative analysis of the intestinal bacterial community in one-to threeweek-old commercially reared broiler chickens fed conventional or antibiotic-free vegetable-based diets. J Appl Microbiol. 2007; 102(4):1138-49.

38. Gong J, Yu H, Liu T, Gill JJ, Chambers JR, Wheatcroft R, et al. Effects of zinc bacitracin, bird age and access to range on bacterial microbiota in the ileum and caeca of broiler chickens. J Appl Microbiol. 2008; 104(5):1372-82. 
39. Pereira R, Bortoluzzi C, Durrer A, Fagundes NS, Pedroso AA, Rafael JM, et al. Performance and intestinal microbiota of chickens receiving probiotic in the feed and submitted to antibiotic therapy. $J$ Anim Physiol Anim Nutr (Berl). 2019; 103(1):72-86.

40. Kumar S, Chen C, Indugu N, Werlang GO, Singh M, Kim WK, et al. Effect of antibiotic withdrawal in feed on chicken gut microbial dynamics, immunity, growth performance and prevalence of foodborne pathogens. PloS one. 2018; 13(2):e0192450.

41. Yegani M, Korver DR. Factors affecting intestinal health in poultry. Poultry Sci. 2008; 87(10):2052-63.

42. Mariat D, Firmesse O, Levenez F, Guimaraes V, Sokol H, Dore J, et al. The Firmicutes/Bacteroidetes ratio of the human microbiota changes with age. BMC Microbiol. 2009; 9:123.

43. Stanley D, Denman SE, Hughes RJ, Geier MS, Crowley TM, Chen H, et al. Intestinal microbiota associated with differential feed conversion efficiency in chickens. Appl Microbiol Biotechnol. 2012; 96(5):1361-9.

44. Gong J, Si W, Forster RJ, Huang R, Yu H, Yin Y, et al. 16S rRNA gene-based analysis of mucosaassociated bacterial community and phylogeny in the chicken gastrointestinal tracts: from crops to ceca. FEMS Microbiol Ecol. 2007; 59(1):147-57.

45. Lu J, Idris U, Harmon B, Hofacre C, Maurer JJ, Lee MD. Diversity and succession of the intestinal bacterial community of the maturing broiler chicken. Appl Environ Microbiol. 2003; 69(11):6816-24.

46. Torok VA, Allison GE, Percy NJ, Ophel-Keller K, Hughes RJ. Influence of antimicrobial feed additives on broiler commensal posthatch gut microbiota development and performance. Appl Environ Microbiol. 2011; 77(10):3380-90.

47. Stecher B, Chaffron S, Kappeli R, Hapfelmeier S, Freedrich S, Weber TC, et al. Like will to like: abundances of closely related species can predict susceptibility to intestinal colonization by pathogenic and commensal bacteria. PLoS 2010; 6(1):e1000711.

48. Bushnell G, Mitrani-Gold F, Mundy LM. Emergence of New Delhi metallo- $\beta$-lactamase type 1producing enterobacteriaceae and non-enterobacteriaceae: Global case detection and bacterial surveillance. J. Infect. Dis.2013; 17: e325-e333.

49. Damborg P, Broens EM, Chomel BB, Guenther S, Pasmans F, Wagenaar JA, et al. Bacterial zoonoses transmitted by household pets: state-of-the-art and future perspectives for targeted research and policy actions. J Comp Pathol. 2016; 155(1): S27-40.

50. Eid S, Samir AH. Extended-spectrum beta-lactamase and Class 1 integrons in multidrug-resistant Escherichia coli isolated from turkeys. Vet World. 2019; 12(7).

51. Friedman M, Henika PR, Mandrell RE. Antibacterial activities of phenolic benzaldehydes and benzoic acids against Campylobacter jejuni, Escherichia coli, Listeria monocytogenes, and Salmonella enterica. J Food Prot. 2003; 66:1811-21.

52. Alkeskas A, Ogrodzki P, Saad M, Masood N, Rhoma NR, Moore K, et al. The molecular characterization of Escherichia coli K1 isolated from neonatal nasogastric feeding tubes". BMC Infect Dis. 2015; 15:449. 
53. Mellata M. Human and avian extraintestinal pathogenic Escherichia coli: infections, zoonotic risks, and antibiotic resistance trends. Foodborne Pathog Dis. 2013; 10(11):916-32.

54. Dziva F, Stevens MP. Colibacillosis in poultry: unravelling the molecular basis of virulence of avian pathogenic Escherichia coli in their natural hosts. Avian Pathol. 2008; 37(4):355-66.

55. Bergeron CR, Prussing C, Boerlin P, Daignault D, Dutil L, Reid-Smith RJ, et al. Chicken as reservoir for extraintestinal pathogenic Escherichia coli in humans, Canada. Emerg Infect Dis. 2012; 18(3):415.

56. Jakobsen L, Spangholm DJ, Pedersen K, Jensen LB, Emborg HD, Agerso Y, et al. Broiler chickens, broiler chicken meat, pigs and pork as sources of ExPEC related virulence genes and resistance in Escherichia coli isolates from community-dwelling humans and UTI patients. Int J Food Microbiol. 2010; 142(1-2):264-72.

57. Rodriguez-Siek KE, Giddings CW, Doetkott C, Johnson TJ, Fakhr MK, Nolan LK. Comparison of Escherichia coli isolates implicated in human urinary tract infection and avian colibacillosis. Microbiol Read Engl. 2005; 151:2097-110.

58. Manges AR. Escherichia coli and urinary tract infections: the role of poultry-meat. Clin Microbiol Infect. 2016; 22:122-9.

59. Mirsepasi H, Persson S, Struve C, Andersen LO, Petersen AM, Krogfelt KA. Microbial diversity in fecal samples depends on DNA extraction method: easyMag DNA extraction compared to QIAamp DNA stool mini kit extraction. BMC research notes. 2014; 7(1):50.

60. Suckling L, McFarlane C, Sawyer C, Chambers SP, Kitney RI, McClymont DW, et al. Miniaturisation of high-throughput plasmid DNA library preparation for next-generation sequencing using multifactorial optimisation. Synthetic and systems biotechnology. 2019; 4(1):57-66.

61. Tawfik SA, Azab MM, Ahmed AA, Fayyad DM. Illumina MiSeq sequencing for preliminary analysis of microbiome causing primary endodontic infections in Egypt. International journal of microbiology. 2018; 2018.

62. Chatterjee A, Rodger EJ, Stockwell PA, Weeks RJ, Morison IM. Technical considerations for reduced representation bisulfite sequencing with multiplexed libraries. BioMed Research International. 2012; 2012.

63. Bolger AM, Lohse M, Usadel B. Trimmomatic: a flexible trimmer for Illumina sequence data. Bioinformatics30, 2114-2120 (2014).

64. Nelson MT, Pope CE, Marsh RL, Wolter DJ, Weiss EJ, Hager KR, et al. Human and Extracellular DNA Depletion for Metagenomic Analysis of Complex Clinical Infection Samples Yields Optimized Viable Microbiome Profiles. Cell reports. 2019; 26(8):2227-40.

65. Bankevich A, Nurk S, Antipov D, Gurevich AA, Dvorkin M, Kulikov AS, et al. SPAdes: a new genome assembly algorithm and its applications to single-cell sequencing. Journal of computational biology. 2012; 19(5):455-77.

66. Buchfink B, Xie $C$, Huson DH. Fast and sensitive protein alignment using DIAMOND. Nature methods. 2015; 12(1):59. 
67. Huson DH, Auch AF, Qi J, Schuster SC. MEGAN analysis of metagenomic data. Genome research. 2007; 17(3):377-86.

68. Danecek P, Auton A, Abecasis G, Albers CA, Banks E, DePristo MA, et al. The variant call format and VCF tools. Bioinformatics. 2011; 27(15):2156-8.

69. Thompson JD, Higgins DG, Gibson TJ. CLUSTAL W: improving the sensitivity of progressive multiple sequence alignment through sequence weighting, position-specific gap penalties and weight matrix choice. Nucleic acids research. 1994; 22(22):4673-80.

70. Criscuolo A., Gascuel O. Fast NJ-like algorithms to deal with incomplete distance matrices. BMC Bioinformatics.2008; 9:166.

71. Breitwieser FP, Salzberg SL. Pavian: Interactive analysis of metagenomics data for microbiomics and pathogen identification. BioRxiv. 2016; 084715.

72. Keohane DM, Woods T, O Connor P, Underwood S, Cronin O, Whiston R, et al. Four men in a boat: Ultra-endurance exercise alters the gut microbiome. Journal of science and medicine in sport. 2019.

\section{Figures}




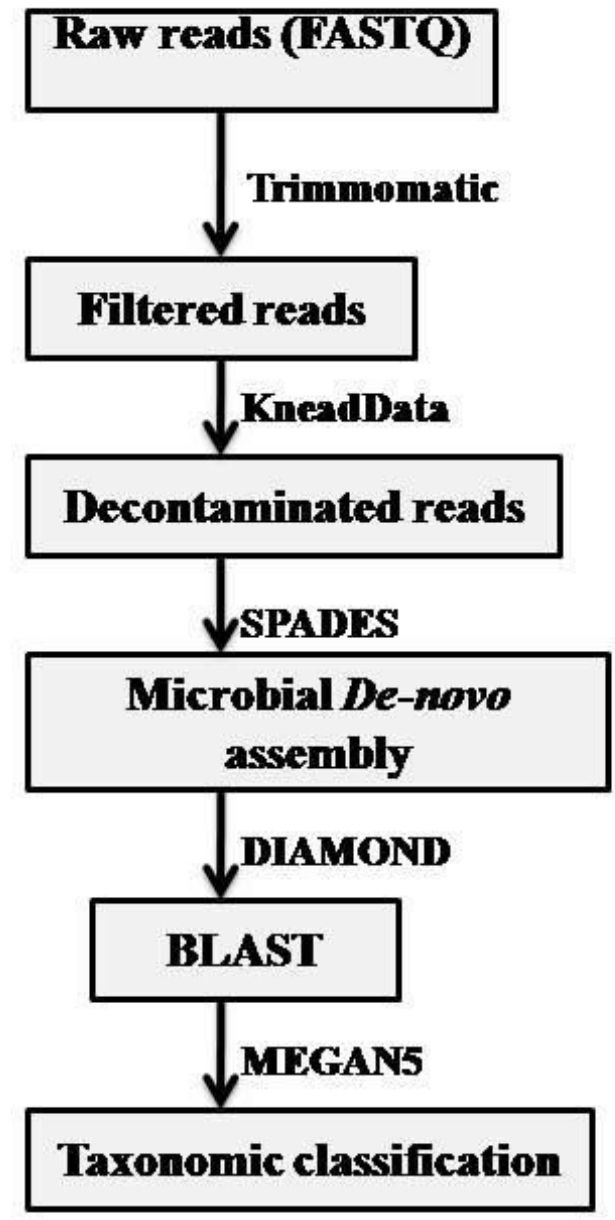

Figure 1

Bioinformatics workflow of our custom data analysis pipeline 


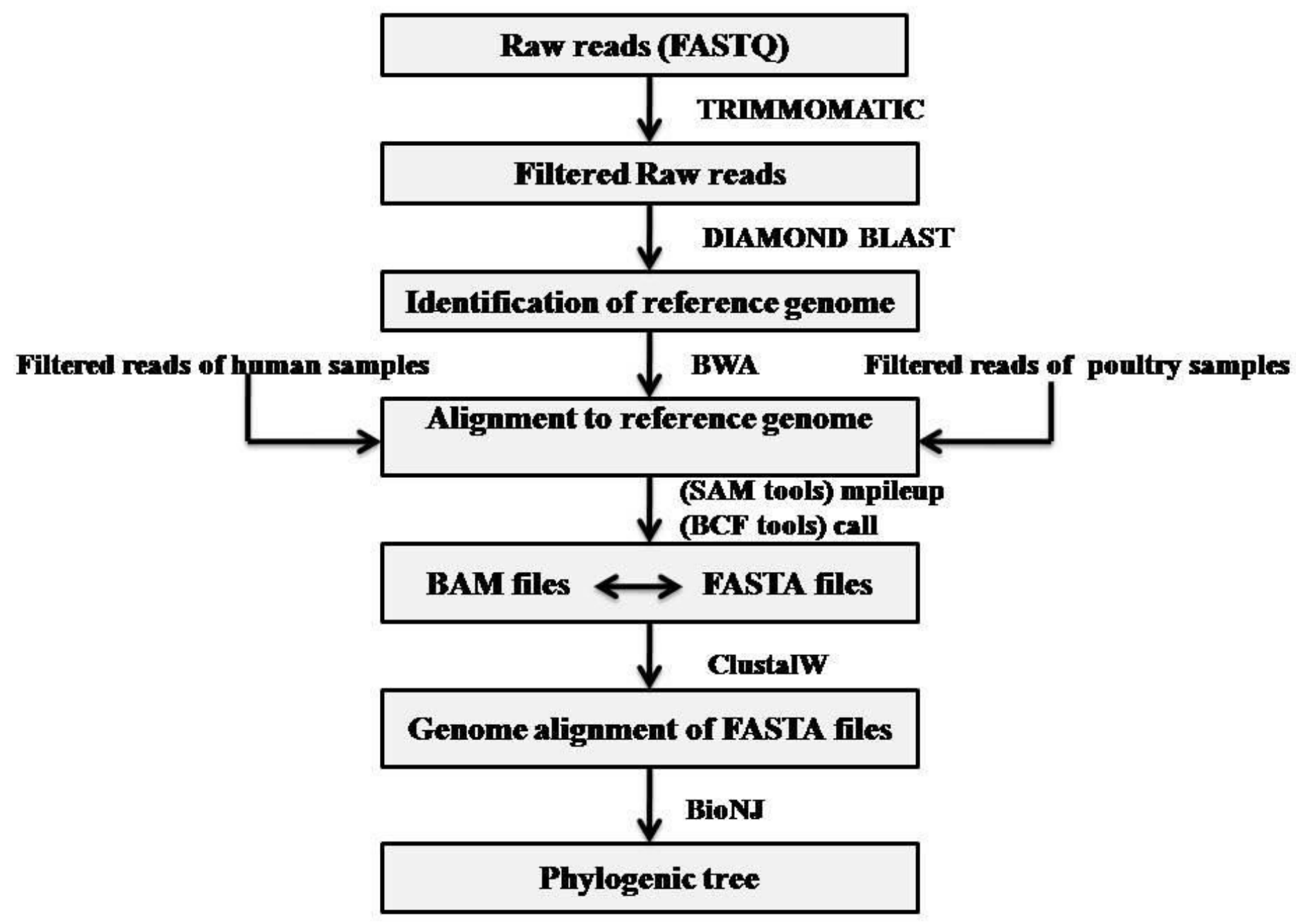

Figure 2

Bioinformatics workflow for the genetic analysis of HS and BC fecal samples with against the reference genome 


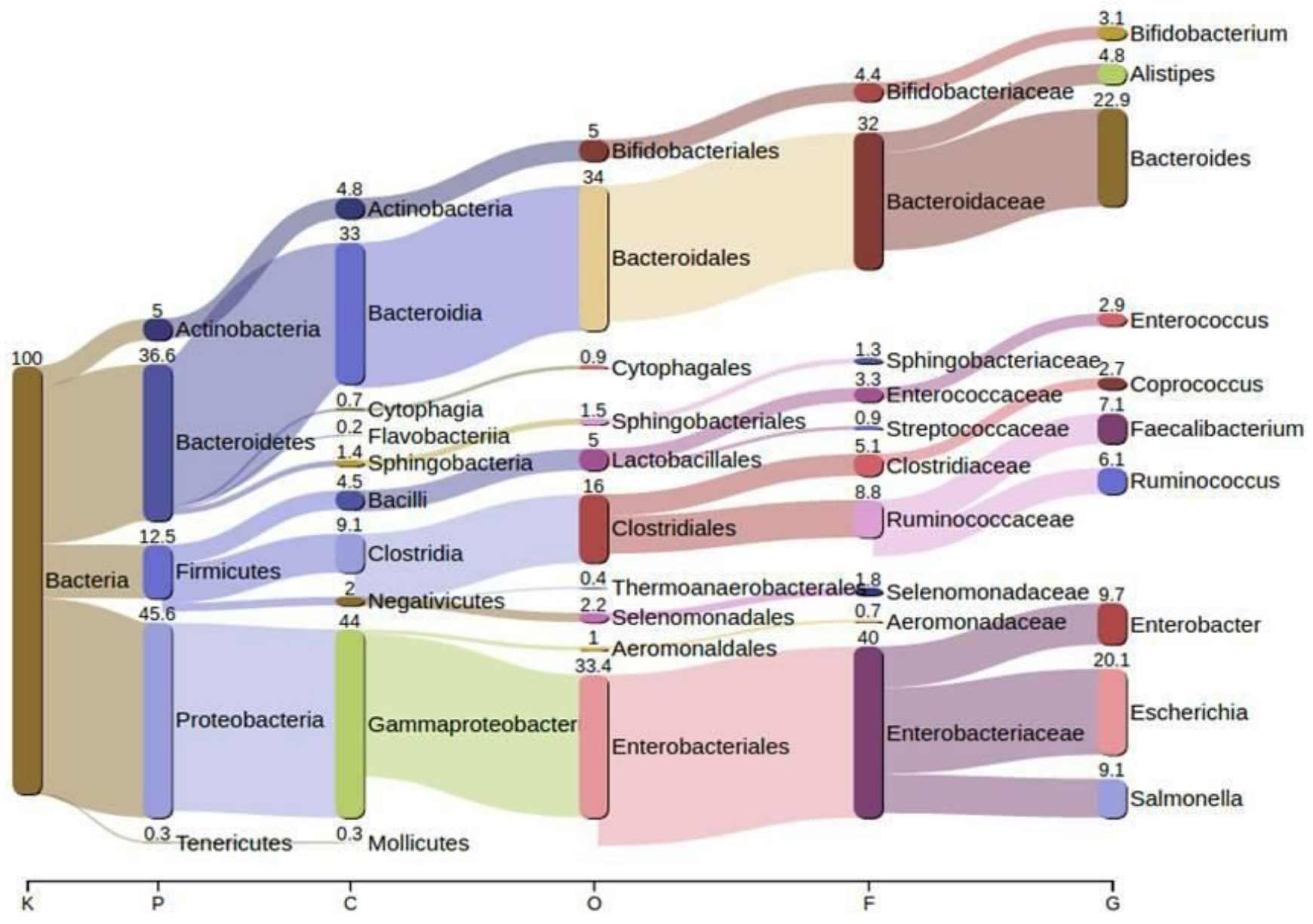

\section{Figure 3}

Taxonomic cladogram showing the bacterial diversity in HS microbiota at various levels using Pavian derived from NGS shotgun metagenomics. Different colored side bars in the Sankey diagram show the relative abundance of bacterial communities in human stool microbiota at various taxonomic levels including kingdom $(K)$, phylum $(P)$, class $(C)$, order $(O)$, family $(F)$ and genus $(G)$. 


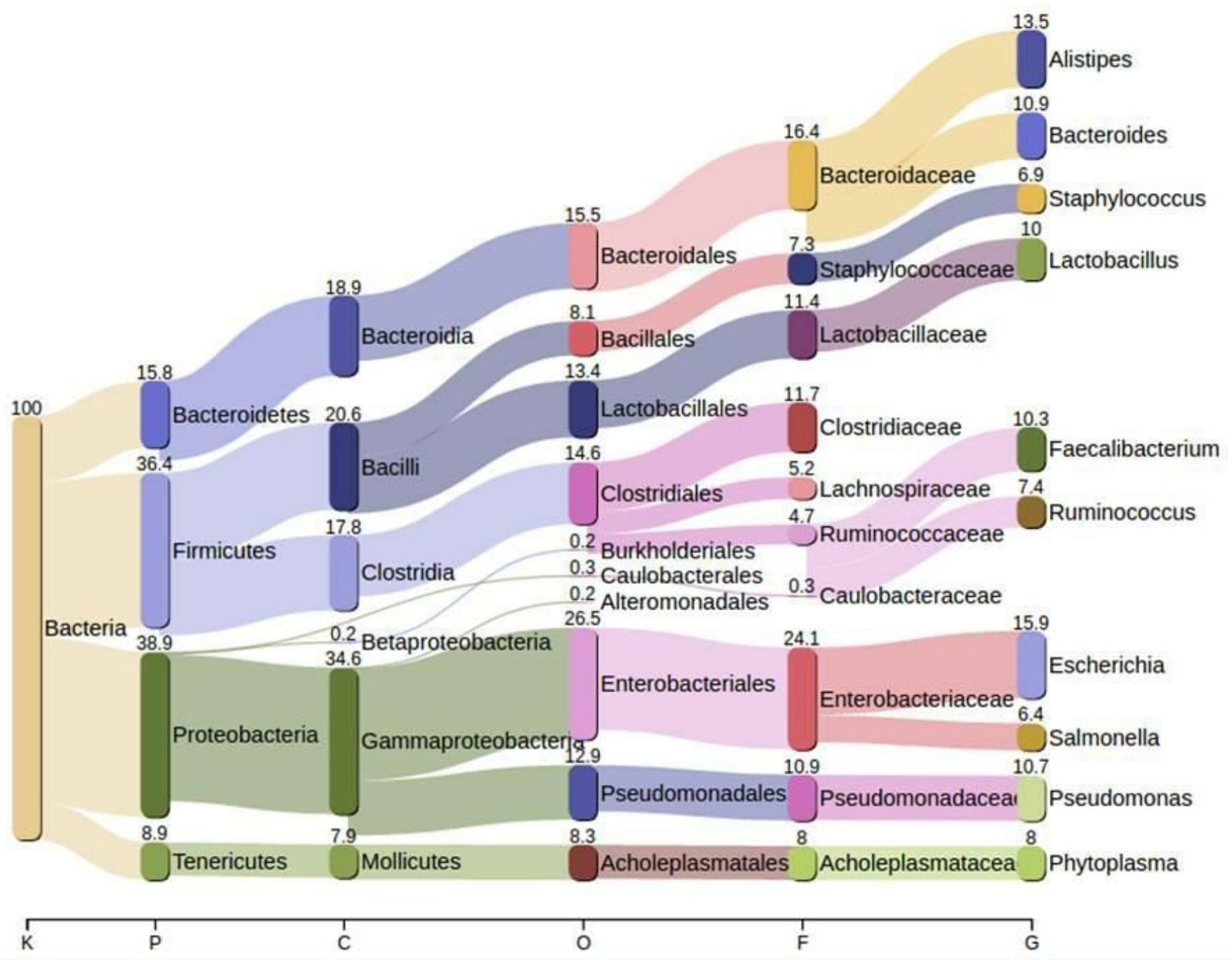

Figure 4

Taxonomic profiling of BC microbiota at various levels using Pavian derived from NGS shotgun metagenomics. Different colored side bars in the Sankey diagram show the relative abundance of bacterial communities in broilers chickens at various taxonomic levels including kingdom (K), phylum (P), class (C), order (O), family (F) and genus (G). 

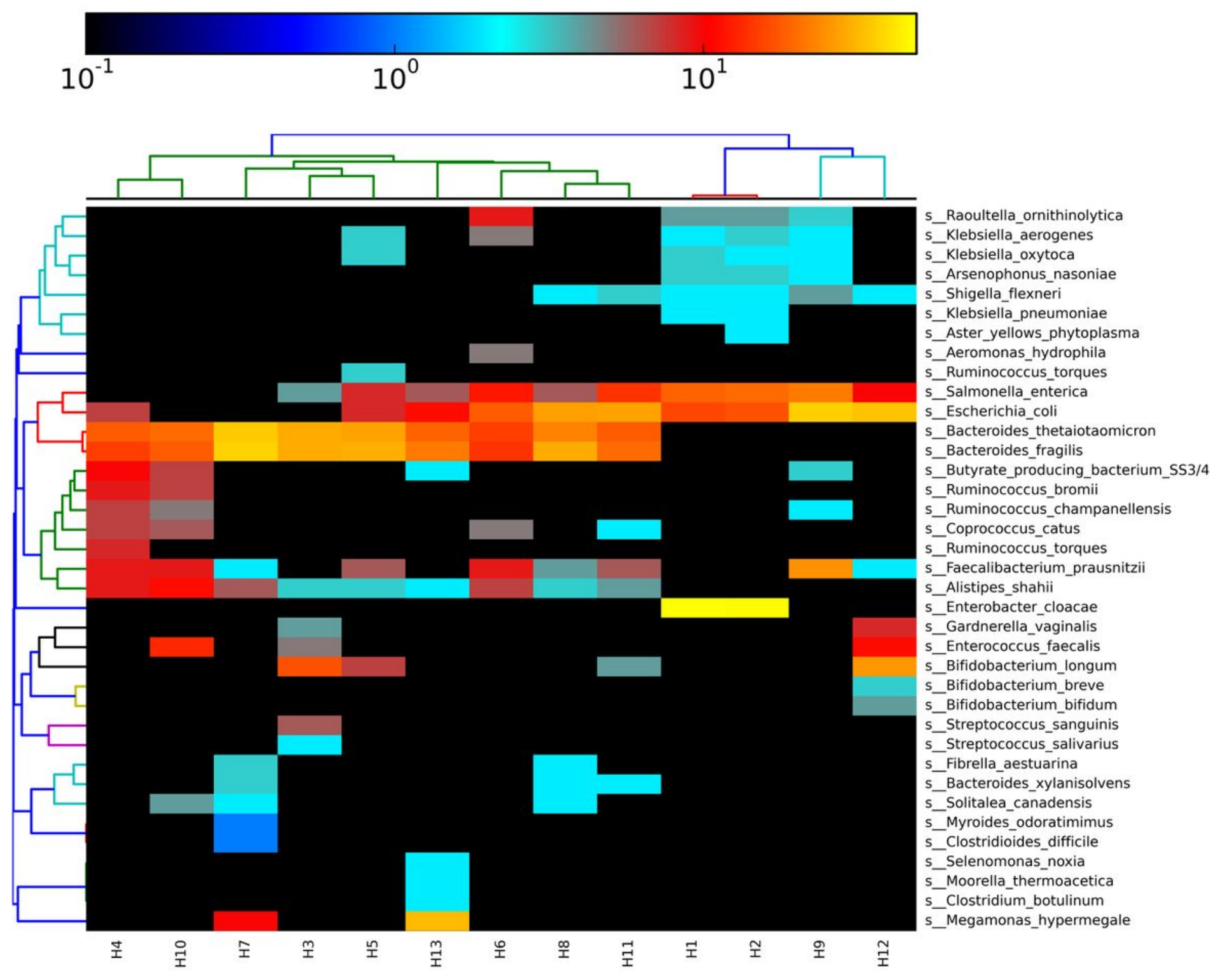

\section{Figure 5}

Heatmap generated through hclust2 showing the abundance of various bacterial species in HS microbiota derived from NGS shotgun metagenomics. Various bacterial species present within the HS microbiota are hierarchically clustered using Bray-Curtis dissimilarity. $\mathrm{H} 1$ to $\mathrm{H} 13$ represents various human stool samples. The relative abundance of various bacterial species is indicated in logarithmic values (base 10). 

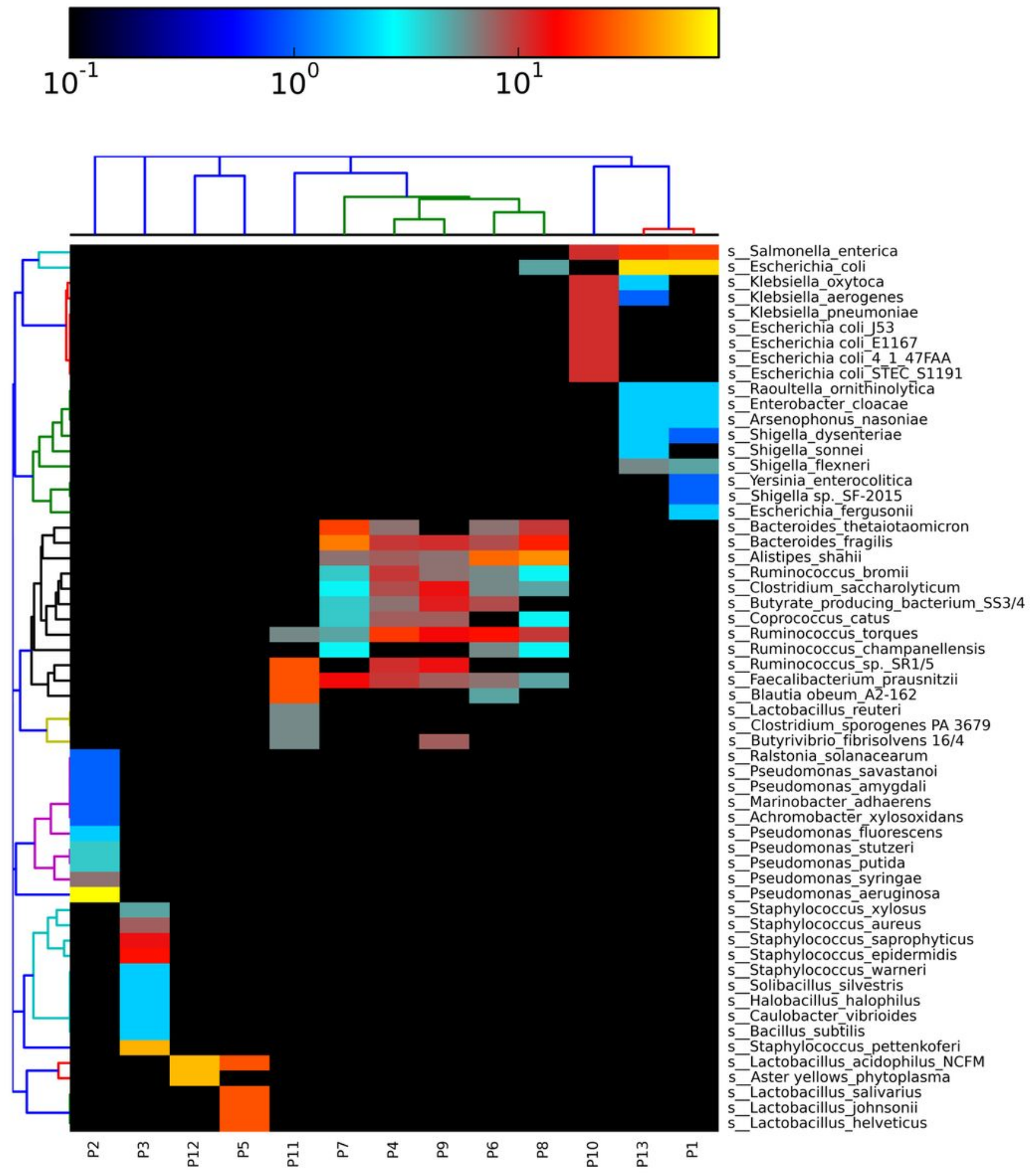

\section{Figure 6}

Heatmap generated through hclust2 showing the abundance of various bacterial species in BC microbiota derived from NGS shotgun metagenomics. Various bacterial species present within the human stool microbiota are hierarchically clusteredusing Bray-Curtis dissimilarity. Where P1 to P13 represents various broiler chicken samples i.e. poultry. The relative abundance of various bacterial species is indicated in logarithmic values (base 10). 

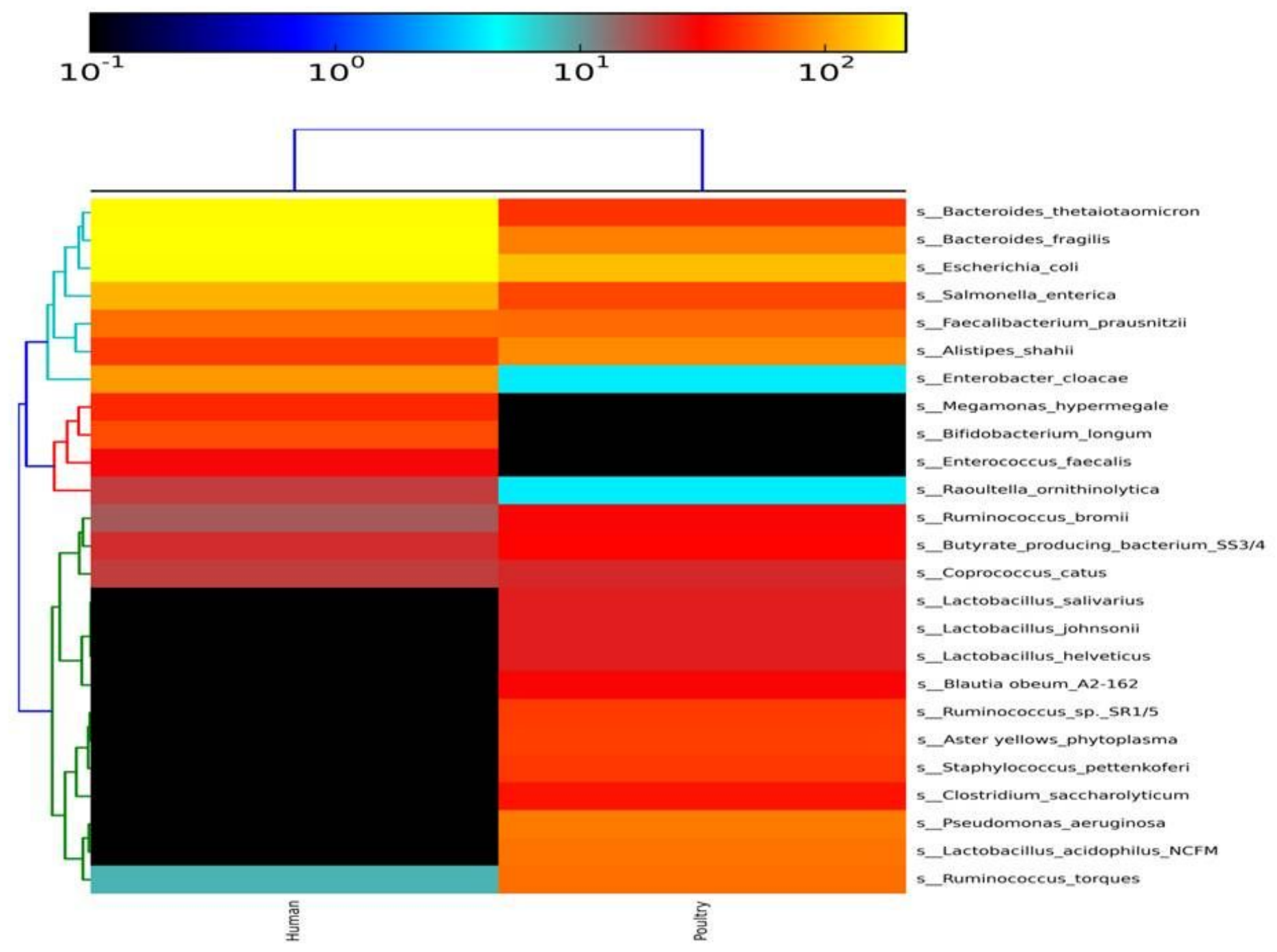

Figure 7

Combined heatmap generated through hclust2 showing the abundance of various bacterial species in $\mathrm{HF}$ and BC microbiota derived from NGS shotgun metagenomics. The diversity of bacterial species present within the human and poultry fecal microbiota are hierarchically clustered using Bray-Curtis dissimilarity. The relative abundance of various bacterial species is indicated by logarithmic values (base 10). 


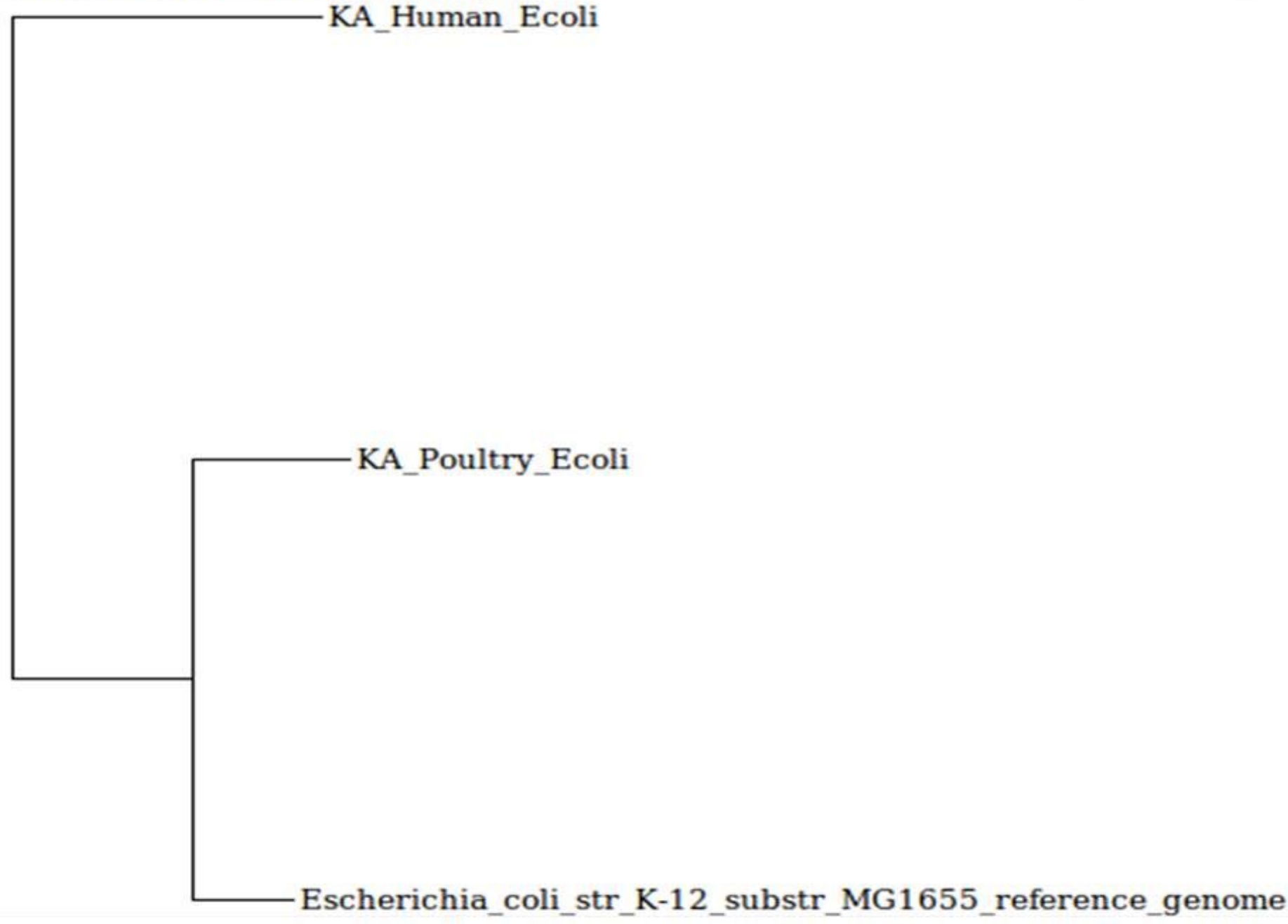

\section{Figure 8}

Phylogentic analysis of human and poultry E.coli against a reference strain E.coli str. K12 MG1655 reference genome. Where KA human E.coli and KA poultry E.coli describes E.coli from HS and BC fecal microbiota respectively. 